\title{
THE GRACE FOLLOW-ON QUIET ELECTRICAL POWER SYSTEM
}

\author{
Manfred Amann ${ }^{(1)}$, Mike Gross ${ }^{(2)}$, Hauke Thamm ${ }^{(3)}$ \\ (1) (3) Airbus Defence and Space GmbH, 88039 Friedrichshafen, Germany, Email: manfred.amann@airbus.com/ \\ hauke.thamm@airbus.com \\ (2) Jet Propulsion Laboratory, California Institute of Technology, 4800 Oak Grove Drive, Pasadena, CA 91109-8099, \\ USA, Email: michael.a.gross@jpl.nasa.gov
}

\begin{abstract}
This paper provides and overview of the GRACE-FO Electrical System and an in depth discussion of the Electrical Power System (EPS). The EPS elements are presented and their features described by their key parameters. These components are inclusive of the body-mounted Solar Array Panels for Power Generation, the Power Control Unit (PCU) and Power Distribution Unit (PDU), a Li-Ion Battery and the Thermal Control System's Heater Control Electronics (HCE's) acting as active current control front-ends. The latter is explained more in detail with respect to its purpose and implementation. The GRACE-FO EPS has been given the designation of "quiet" EPS due to the function of the HCE within the thermal control Subsystem.

The final portion of the paper provides information about the power and energy budgeting and operational aspects of the EPS such as undervoltage and overcurrent $\mathrm{H} / \mathrm{W}$ features of the PCU/PDU design, and the EPS related On-board Software and On-Board Control Procedures. Finally, the system level power analysis is provided to provide the reader with a better understanding of the expected behaviour of the electrical power parameters.
\end{abstract}

\section{SCOPE}

Expected to launch in 2017, the new GRACE-FO Mission* satellite pair will continue and expand upon the measurements initiated by the pathfinder GRACE Mission, launched in 2002. The new mission's primary objective is to estimate the global high-resolution models of the Earth's gravity field for an additional period of five years at a precision and temporal sampling equivalent to that achieved with the original GRACE satellites by means of a microwave interferometer instrument. A second objective is to demonstrate low Earth orbit interferometry by an intersatellite laser ranging interferometer instrument [1][2]. In addition to the accommodation of the instruments described above, the satellites also carry science
Accelerometer Instruments, which are used to determine the non-gravitational forces experienced by the satellites. This instrument suite requires a spacecraft that provides a "quiet" environment for the system to meet its performance requirements. This "quiet" environment includes extreme temperature stability, high mechanical stability, precise alignment, the elimination of platform generated micro-seismic activity and excellent EMC performance.

See an artwork of the GRACE-FO satellites below in figure 1 .

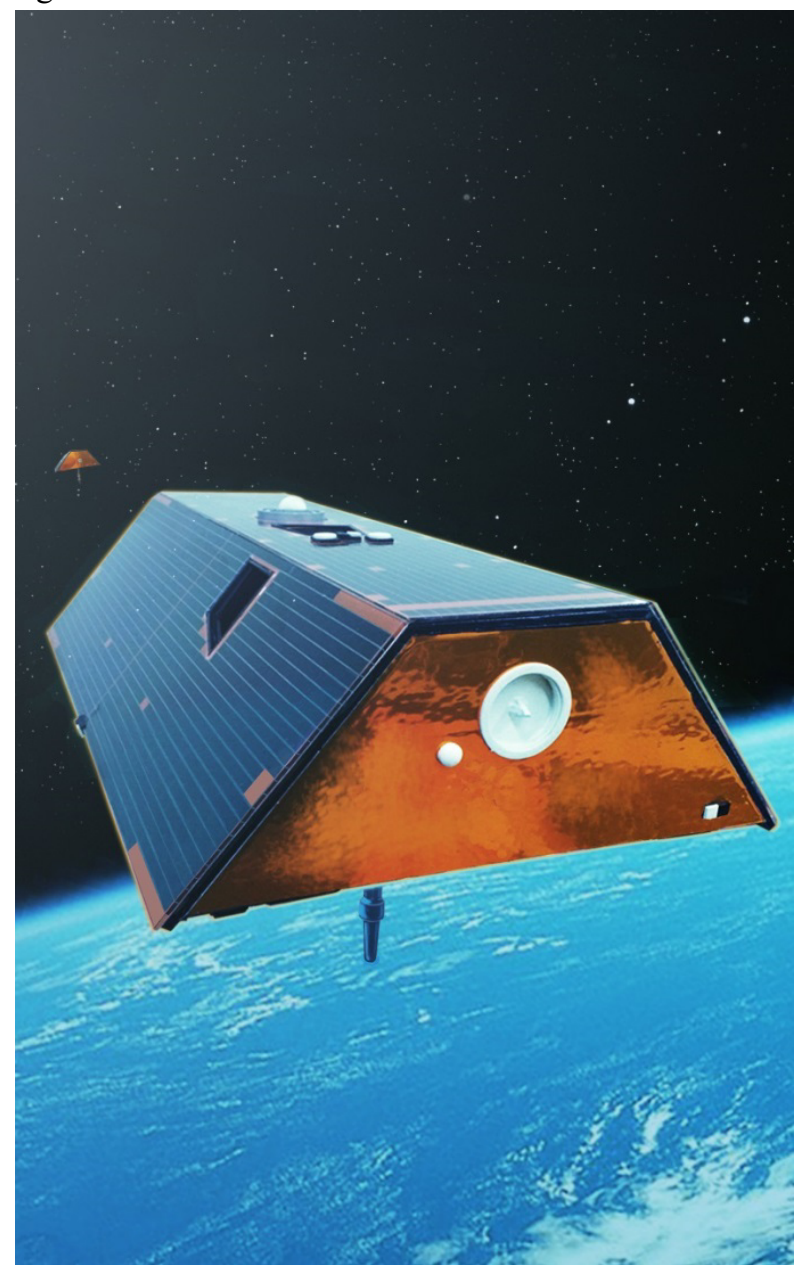

Figure 1: GRACE-FO Satellite constellation artwork Courtesy of JPL 


\section{SATELLITE ELEKTRICAL SYSTEM \\ OVERVIEW}

The GRACE-FO Electrical Block Diagram is depicted hereafter.

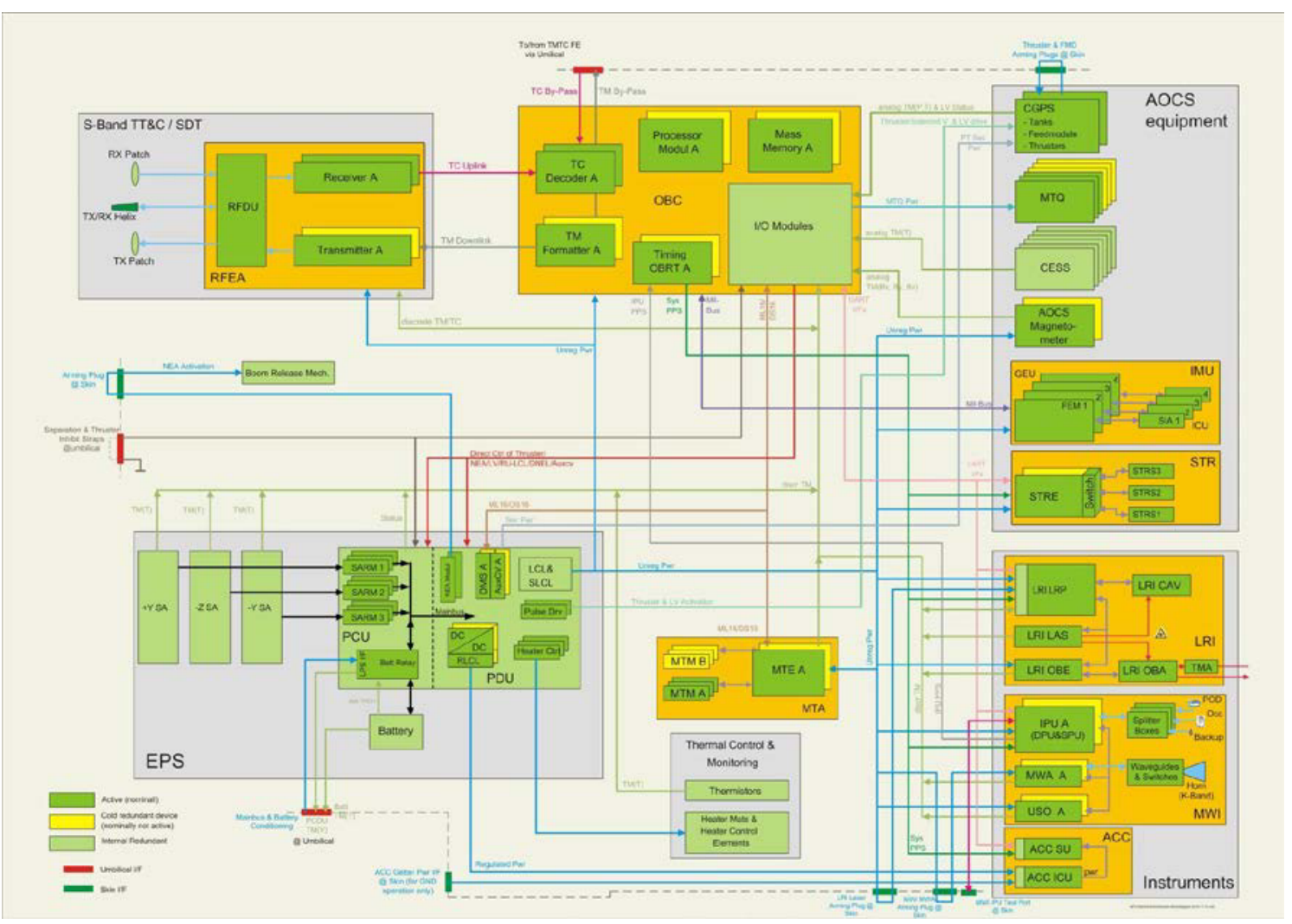

Figure 2: GRACE-FO Electrical Block Diagram

The main blocks are:

- Electrical Power Subsystem (EPS), described hereafter.

- On Board Computer (OBC), the platform data handling system (DHS).

- Telemetry and Telecommand (TT\&C) and Science Data Transmission (SDT) subsystem as interface between the ground station and the $\mathrm{OBC}$ for reception of telecommands and telemetry

- The Attitude and Orbit Control System (AOCS) units ensure the $\mathrm{S} / \mathrm{C}$ attitude and orbit control

- Thermal Control Subsystem

- The GRACE-FO instruments, namely the Laser Ranging Instrument (LRI), the Microwave Instrument (MWI) instrument and the Accelerometer (ACC)

\section{EPS DESIGN}

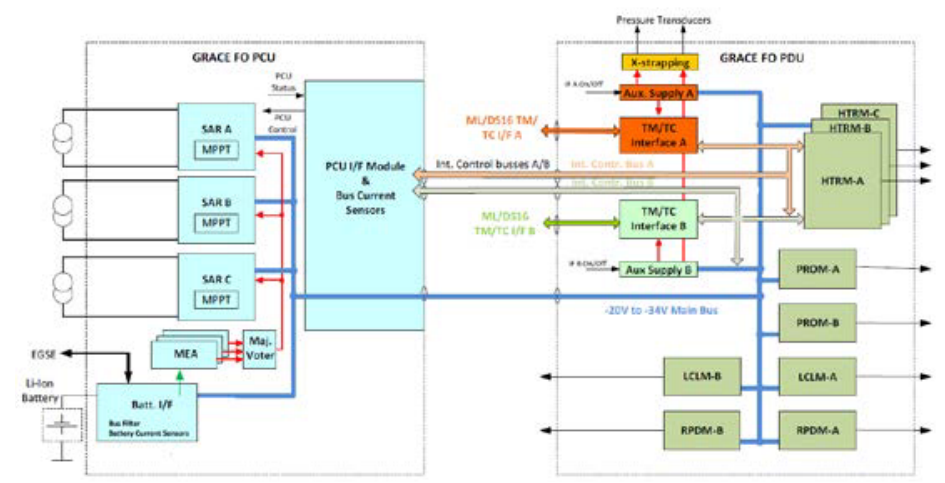

A more detailed block diagram of the GRACE-FO EPS is shown in figure 3 .

Figure 3: EPS Blockdiagram

It shows the 3 main blocks,

- The Solar Generator, consisting of 3 body- 
mounted Solar Panels

- The Battery

- The Power Control and Distribution Unit (PCDU) as central power conversion and power control unit

The PCDU is split into two boxes for accommodation reasons, the Power Control Unit (PCU) and the PDU (Power Distribution Unit).

A GRACE-FO specific feature is the positive grounding of the primary power system. Hence, the power bus is negative with respect to the $\mathrm{S} / \mathrm{C}$ ground. This implies a dedicated design for the primary power conversion from the Solar Array to the PCDU and to the power users.

The reason for this solution is the advantage of reduced $\mathrm{S} / \mathrm{C}$ charging [3] and also heritage from previous missions (original GRACE, CHAMP, SWARM).

\subsection{Solar Array Design}

The Solar Array consists of three body mounted panels, each side panel with 29 strings/combined in 5 sections and the top/zenith panel with 20 strings combined in 4 sections. Each string is equipped with 24 SCAs (Solar Cell Assemblies from AZUR SPACE) of a GaAs triple junction technology with integrated shunt diode. The $\mathrm{S} / \mathrm{C}$ panels' orientation is optimised for the best energy harvest one can get with the given orbit.

A specific characteristic of the GRACE-FO structure is the solar (and rear/front) panel lay-up. For the mechanical stiffness a conventional CFRP honeycomb panel is used, however, on top a highly thermally isolating foam layer of $40 \mathrm{~mm}$ thickness is implemented. The outermost panel layer is a CFRP face sheet. This setup provides excellent thermal isolation for the inner $\mathrm{S} / \mathrm{C}$ compartments. On the plain solar panels this lay-up supports the SCAs' temperatures to be almost equal across all of the individual panels. This is important for the proper operation of the MPPT control scheme of the PCU.

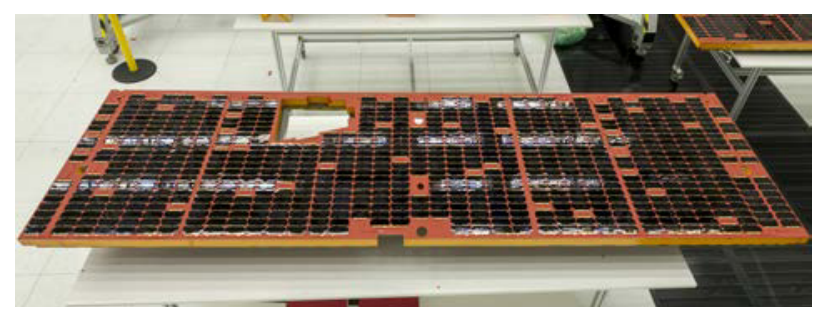

Figure 4 GRACE-FO Side Panel

\subsection{Battery Design}

The GRACE-FO Batteries are equipped with Li-Ion cells with a total nameplate capacity of $78 \mathrm{Ah}$. The Battery configuration is a $8 \mathrm{~s} 52 \mathrm{p}$ ( 8 cells per string and 52 strings in parallel) in a flat single module. See figure 5 below. A specific characteristic of the Battery thermal design is that it is mainly based on radiative coupling to the external environment. It is also equipped with internal heaters and HCEs on the top cover. There are two (plus two redundant) independent heater control loops available to stabilize the Battery cell temperature. (See detailed HCE approach in section 4)

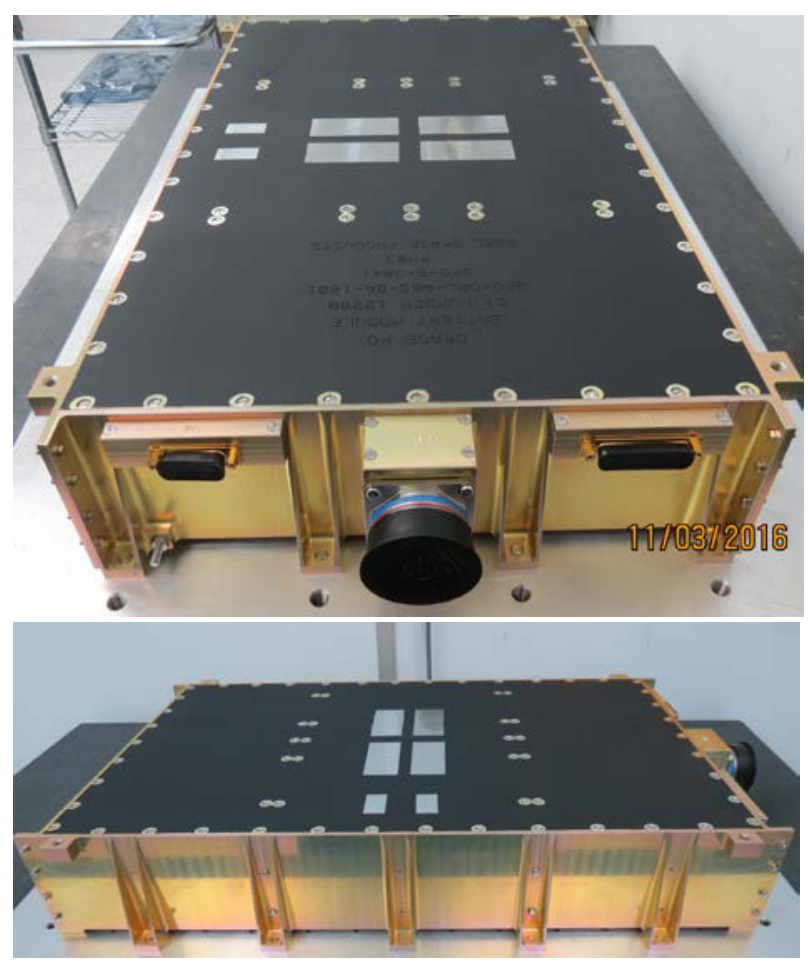

Figure 5 GRACE-FO Flight Battery

\subsection{PCDU Design}

This section lists the PCDU Modules (as shown in figure 3) and provides details regarding the number of channels, the types of outlets and special function incorporation such as battery passivation and regulated outputs. The GRACE-FO PCDU provides a nonregulated power bus (BNR), hence the users have to be tolerant to power bus voltage variations of about $+/-30$ $\%$. The PCDU is split in two physical housings, for accommodation reasons.

Find below the PCU/PDU assembly during EMC Testing. 


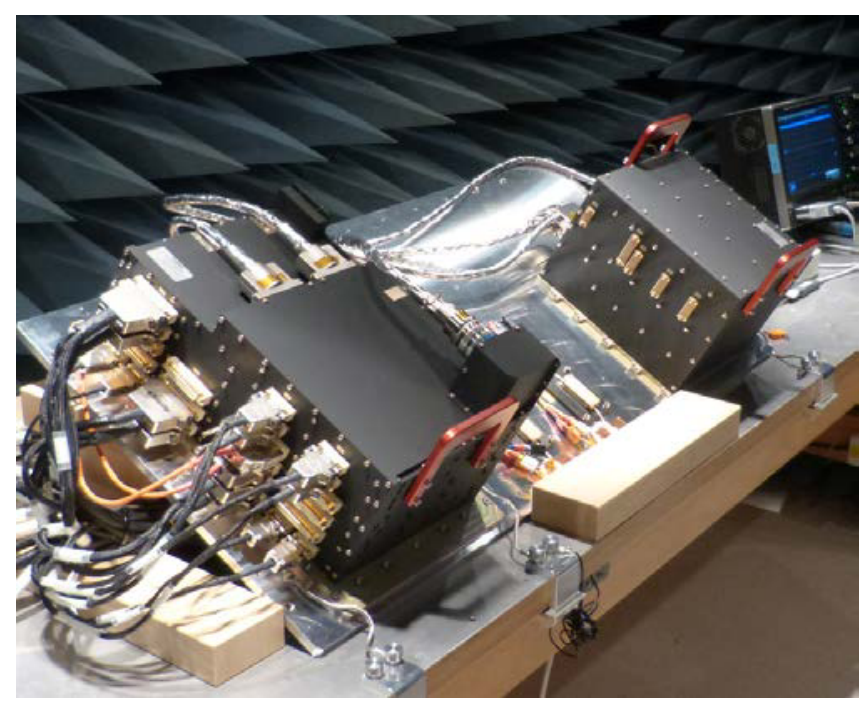

Figure 6 PCU and PDU in EMC Test Chamber

\subsubsection{PCU Design}

The PCU consists of 6 modules, which are

- 3 SARM (Solar Array Regulator Module), one for each Solar Array Panel; each SARM consists of 3 independent SARs for redundancy reason; 2 out of the 3 are able to convert the full power required

- Main Error Amplifier Module, which controls the SARM power to adjust the battery charging current and hosts the power bus voltage underand overvoltage protection

- Battery I/F Module with a capacitor bank to stabilize the power bus, Battery relays and power bus current sensors

- PCU Interface Module, which provides the power and signal interfaces to the PDU and contains the Battery passivation circuitry. The passivation function works such that it disables the SARM, which leads to an overdischarge of the battery and the depletion of the electrical energy of the system.

\subsubsection{PDU Design}

The PDU part of the PCDU contains the modules:

- Redundant Latching Current Limiter Modules (LCLM), each with 11 outlets, which supply most of the platform units from the unregulated power bus
- Redundant Regulated Power Distribution Modules (RPDM). Each RPDM carries 7 outlets to supply the payload units (one with regulated power) and an actuator channel

- Redundant Propulsion Modules (PROM), each carrying 7 thruster, 6 Latch Valve and one Solenoid Valve drive channel

- Redundant Data Management System Modules (DMSM), which serve for interfacing to the OBC and for the generation of auxiliary voltages for the other PDU and PCU modules

- PDU Interface Module to manage the interfaces to the PCU.

\section{THERMAL CONTROL SYSTEM (TCS) OF GRACE-FO}

Due to the very demanding temperature stability requirements ("quiet" $\mathrm{S} / \mathrm{C}$ ) on the instrument temperatures and mechanical stability requirements, very high attention has been paid to the GRACE-FO TCS and mechanical design. Passive measures are implemented such as

- $\quad$ Structure lay-up with foam layers

- Low CTE selection of structure material

- strong thermal isolation where temperature changing environment is in the view of a thermally sensitive unit or a conductive path is available to a thermally varying element

- $\quad$ Nadir side thermal radiators

In addition complex active temperature stabilisation has been established

\subsection{Thermal Control Software and Algorithms}

All heater loops are S/W controlled and are individually configurable by parameters to PI control algorithms. The algorithms calculated power demand is realised by 7 bit resolution heater current adjustment of the dedicated channels. To avoid high power dissipation in the PDU and to capture waste heat from the current regulation, a byproduct of the chosen linear regulation scheme, all heater power stages are located next to their designated heaters. The implemented algorithm also allows for some adjustments and settings which are

- adjustable assignment of any on-board thermistor reading for the control loop's actual temperature

- $\quad$ single point failure free triple majority voting of thermistor readings with adjustable criteria 
- for more accurate power control, the mainbus voltage can be considered in the algorithm

- adjustable maximal allowed current step size to limit the rate of current change and reduce bus load variations

- total current monitoring of heater groups to safeguard the EPS in case of a single channel malfunction and to avoid excessive power consumption

\subsection{Heater Control Element Approach}

As mentioned above, each heater channel is a combination of three elements; the On Board Computer (TCS algorithm and telemetry monitoring), the PDU (distribution of primary power and channel current fault protection, and the HCE itself. The core of each HCE is the "linearly" controlled power stage (MOSFET), which allows the TCS to recapture waste heat of the current regulation operation due to its integration alongside the TCS heaters that it regulates current to. Find a picture of an HCE unit in figure 6. The PDU provides 72 (3 modules by 24) channels. This high number of heater channels allows placing heaters and the related HCE's at many locations instead of only a few, and by design, keeps thermal control zones stable, thus minimizing the thermal disturbances in the system. This combination of HCE application with its linear control across the spacecraft system provides for the "quiet' nature of the spacecraft, which has been discovered to be necessary on both the Champ and GRACE Missions. The STAR and SuperSTAR Accelerometers have, in flight, measured micro-seismic events originating from the typical thermostatically controlled TCS. The historic "bang-bang" control associated with thermostats generates mass displacement and recoveries in the spacecraft structure that theorized and analysed to be due to both thermal distortion and Lenz law interactions with the structure.

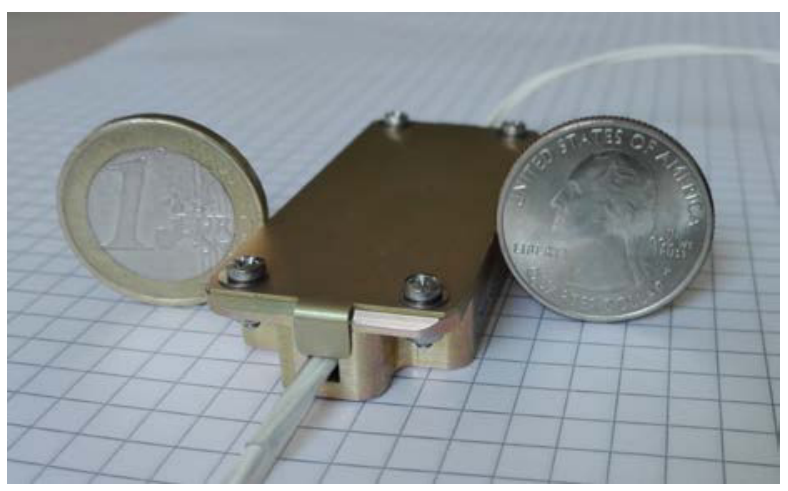

Figure 7 HCE unit

\section{POWER AND ENERGY BUDGET}

\subsection{On-board Power Demand}

The power budget has been set up to service the power demand of the S/C power users in the different orbit scenarios and different mission phases. The expected worst case power has been calculated to be approx. 280 W.

A considerable amount is assigned to heater power, which is by nature the biggest uncertainty in most satellites.

\subsection{Power Generation}

The EPS relevant GRACE-FO Mission and orbit parameters are:

- $\quad$ LEO (Low Earth Orbit) between 490km (BoL) and $410 \mathrm{~km}$ (EoL).

- High inclination angle (close to polar orbit), which imposes a slow drifting orbital plane and a high percentage of orbits with eclipses.

- Satellites shall always be nadir oriented.

- $\quad$ Each satellite may fly forward or backward.

- Body mounted Solar Arrays

These parameters imply a complex analysis of different cases for power generation and thermal conditions.

The analysis has been performed by the Airbus DS satellite power tool Power Systema [4] with the model as shown below in figure.

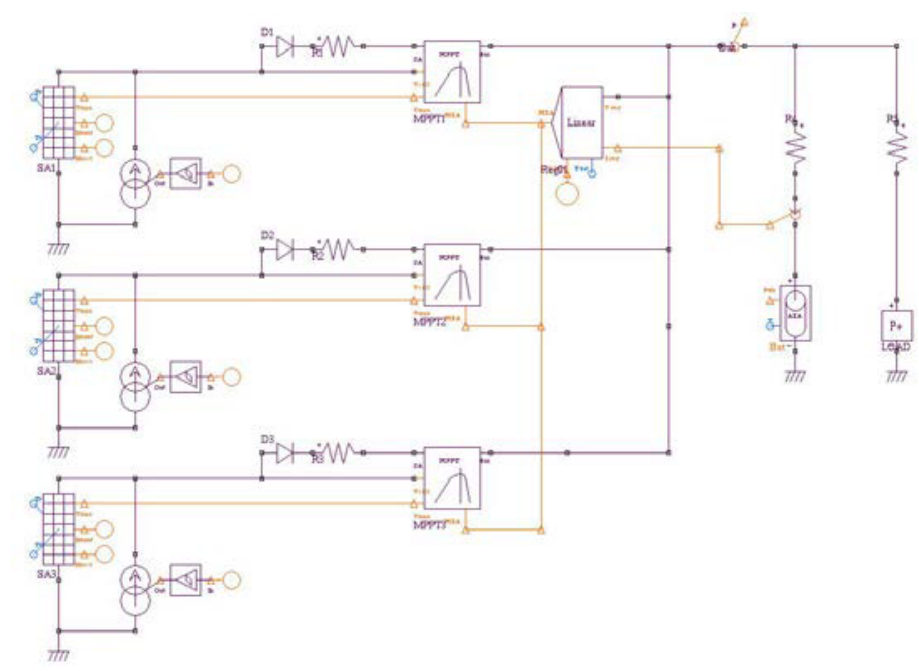

Figure 8 Power Systema model for GRACE-FO

For the above mentioned conditions the possible average power per orbit under worst case conditions has been analysed for each of the 24 LTAN hours. The 
results are shown in the following profile chart (average values per orbit):

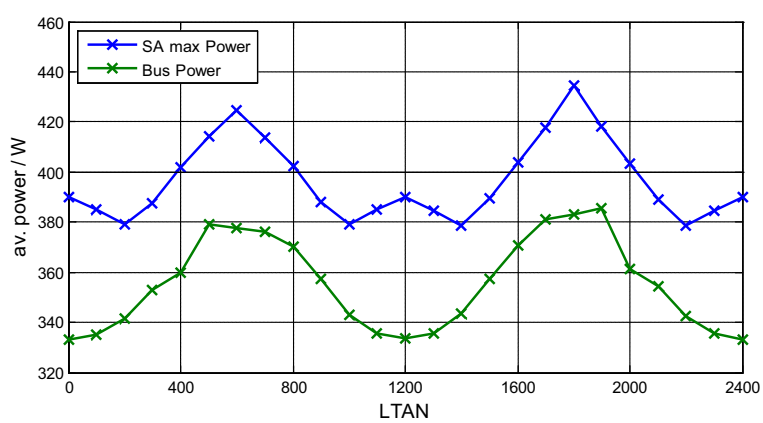

Figure 9 GRACE-FO energy gneration dependency on LTAN

This was the first step before other cases were studied with modified conditions and configurations such as load variations, mission phases, equipment ageing, etc.

These cases were analysed mainly by time domain simulations with 5 to 10 orbits to be able to interpret the results correctly.

An example of a plot set at EoL is shown below in figure 10 .

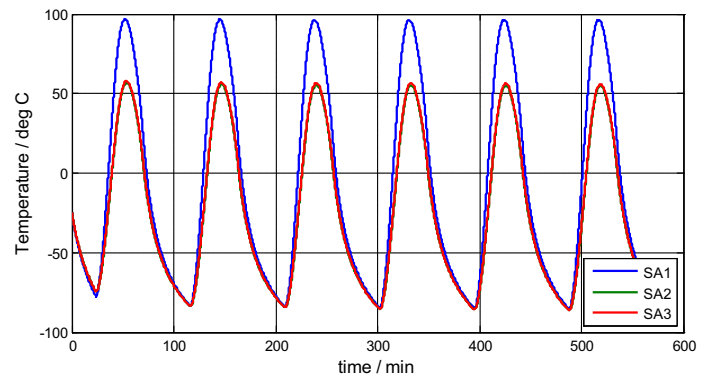

Solar Array temperatures (SA2 and SA3 are identical)

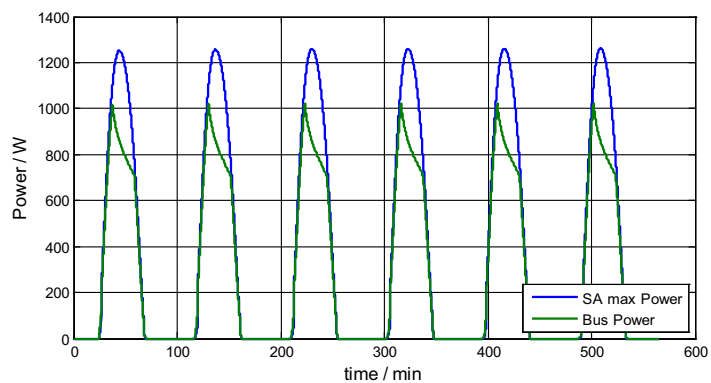

Solar Array max power capability and the used power for the power bus

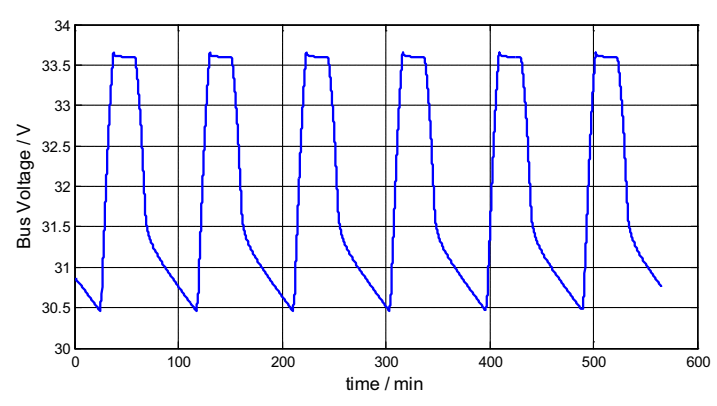

Power Bus voltage

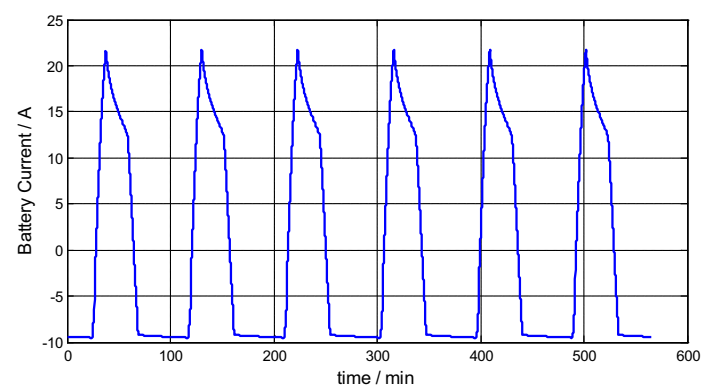

Battery current

Figure 10 Power Systema simulation for an LTAN1200 orbit

\subsection{Power System Contingency Design}

This section describes features of the GRACE-FO H/W measures in case of contingency cases, in addition to the current limiters at the PDU outputs. It includes also the On-Board Control Procedures (OBCP) implementation to ensure a protected power system operation on the $\mathrm{S} / \mathrm{C}$.

The PCU provides as H/W protection features:

- Battery relay hold current capability to ensure closed battery relays during launcher ascent

- Battery charge and discharge current limitation

- Solar Array Regulator switch-off and interlocking

The PDU provides as $\mathrm{H} / \mathrm{W}$ protection features

- Disable Non-Essential Loads (DNEL) logic in case of unexpected low mainbus voltage

- Aux Converter switch-off interlocking

- Current limiter output stage protection in case of load oscillation

The Failure Detection and Isolation Recovery (FDIR) concept of the GRACE-FO Platform supports the PCDU $\mathrm{H} / \mathrm{W}$ detection and serves for additional detection measures by monitoring the on-board telemetry (TM). 
EPS relevant FDIR cases are:

- Mainbus voltage monitoring leading to a S/C mode transition in case the mainbus drops under a threshold value

- Supply current monitoring of outlet currents and off commanding when upper and/or lower current values are exceeded.

- Temperature monitoring can be configured to lead to switch the heater loop to the redundant channel

- $\quad$ Any other TM parameter can be used to trigger an on-board control procedure

\section{CONCLUSION}

The GRACE-FO Electrical Power System has been designed and optimized to fulfil the challenging mission requirements on the $\mathrm{S} / \mathrm{C}$ platform. The EPS units/equipment with their key figures have been presented. By means of the HCE approach, the EPS provides the functionality for the complex, configurable, efficient and "quiet" Thermal Control System. The TCS Software allows the system to benefit from the multiple adjustment features.

Finally the EPS power analysis has been exemplarily presented and the $\mathrm{H} / \mathrm{W}$ and $\mathrm{S} / \mathrm{W}$ supported contingency functionality has been explained.

\section{ACKNOWLEDGEMENTS}

The work described in this paper was carried out at Airbus Defence and Space, GmbH, under contract to the Jet Propulsion Laboratory, California Institute of Technology. The Jet Propulsion Laboratory, California Institute of Technology is managing the GRACE-FO Project development under a contract with the National Aeronautics and Space Administration. US Government sponsorship acknowledged.

\section{REFERENCES}

[1] http://gracefo.jpl.nasa.gov/mission/

[2] http://www.gfz-potsdam.de/en/section/globalgeomonitoring-and-gravityfield/topics/development-operation-and-analysisof-gravity-field-satellite-missions/grace-fo/

[3] Solar Array Arcing Mitigation for Polar LowEarth Orbit Spacecraft, Grant Bonin et al., UTIAS Space Flight Laboratory

[4] Investigation results on solar array thermal and electrical imbalance phenomenon on power systems equipped with MPPT, F. Mercier et al., ESPC2014 paper 